\title{
SORAFENIB, RISK OF BLEEDING AND SPONTANEOUS RUPTURE OF HEPATOCELLULAR CARCINOMA. A CLINICAL CASE
}

\author{
Ferdinando Rombolà', Angelo Caravetta ${ }^{2}$, Francesco Mollo ${ }^{3}$, Antonio Spinoso ${ }^{4}$, Lenino Peluso $^{3}$, Raffaele Guarino $^{2}$
}

Emergency Medicine Unit, "Jazzolino” Hospital, Vibo Valentia, and Postgraduate Internal Medicine School, Faculty of Medicine, University of Messina, Italy"; General Surgery Unit, "Beato Angelo" Hospital, Acri, Cosenza, Italy²; Emergency Unit, "Beato Angelo" Hospital, Acri, Cosenza, Italy3; Oncology Unit, ASP Vibo Valentia, Italy"

Summary: Spontaneous rupture is a rare and dramatic complication of hepatocellular carcinoma (HCC), burdened by a high mortality. Here we describe a case of a 73-year-old man, who arrived at the ER because of syncope, and acute epigastric and right upper quadrant abdominal pain. He had a history of hepatitis C-related liver cirrhosis and HCC in treatment with sorafenib. The physical examination showed a state of hemorrhagic anemia with the presence of blood in the peritoneal cavity. The patient underwent an urgent liver resection. Thirty days after surgery, he was in good general condition. Sorafenib is a multikinase inhibitor recently introduced in the therapy of patients with advanced HCC. Among the various side effects reported in patients treated with sorafenib, there is a higher risk of bleeding. In conclusion, sorafenib may increase the risk of bleeding and rupture of HCC in susceptible individuals.

Key words: Hepatocellular carcinoma; HCC; Rupture; Bleeding; Sorafenib; Side effects

\section{Introduction}

Sorafenib is a multikinase inhibitor of the vascular endothelial growth factor (VEGF) pathway recently introduced in the therapy of advanced hepatocellular carcinoma. Treatment with sorafenib is associated with a significant increase in the risk of bleeding. This report describes the case of a large hepatocellular carcinoma, bleeding from a spontaneous rupture, in a patient taking sorafenib.

\section{Case Report}

A 73-year-old Caucasian man came to the emergency room because of syncope and acute epigastric and right upper quadrant abdominal pain. He had a history of hepatitis C-related liver cirrhosis and HCC, in treatment with sorafenib $400 \mathrm{mg}$ orally twice daily. On examination, the patient looked pale and sweaty. The temperature was $36.5^{\circ} \mathrm{C}$, blood pressure $90 / 70 \mathrm{mmHg}$, heart rate 110 beats/minute. The abdomen was slightly distended, with tenderness and rebound tenderness in epigastrium and right upper quadrant. The hematocrit was $29.2 \%$ (normal range 36-54\%), hemoglobin $9.2 \mathrm{~g} / \mathrm{dl}$, platelets $371.000 \mathrm{mmc}$, total bilirubin $1.17 \mathrm{mg} / \mathrm{dl}$, prothrombin time $78 \%$, INR 1.16, albumin $2.37 \mathrm{~g} / \mathrm{dl}$, creatinine $1.1 \mathrm{mg} / \mathrm{dl}$. Ultrasound-guided diagnostic paracentesis showed blood in the peritoneal cavity. The patient had a previous abdominal CT scan, performed 5 days before, which revealed a large hepatic tumor of $12.8 \mathrm{~cm}$ diameter, and corpuscular fluid around the liver (Fig. 2). The patient, with a Child-Pugh score of B7 and a MELD score of 10, underwent to atypical liver resection, including the tumor mass. The postoperative course was regular and the patient was discharged after 9 days. Thirty days after surgery, he was in good general condition.

\section{Discussion}

Each year in the world, there are more than 626.000 new cases of HCC. Its incidence is increasing in Europe and the United States of America (1). The spontaneous rupture of $\mathrm{HCC}$ is a rare but dramatic complication. Its incidence is around $7-11.2 \%$, according to the geographic area, and is greater in endemic areas for hepatitis B and C, especially in the East (2-4). It is burdened by high mortality, which in 30 days, range from 32 to $67 \%(2,5,6)$. Risk factors, with increased rate of mortality, are a high Child-Pugh score, high levels of serum bilirubin, hepatic encephalopathy and hypoalbuminemia $(2,6,7)$. Therefore, a poor hepatic functional reserve is associated with a poor prognosis. The causes of spontaneous rupture of HCC are unclear. It has been shown that a large tumor, localized at the surface of the liver, is associated with increased risk of rupture (8). Clinical presentations of HCC rupture is the sudden onset of abdominal pain, hypotension and abdominal distension. The next steps in diagnosis are abdominal ultrasound, diagnostic paracentesis and abdominal CT scan (9). The diagnosis of rupture can be difficult, especially in patients with no history of HCC or liver disease (10). In most cases, the sudden onset of abdominal pain, and hemoperitoneum, in the presence of HCC, can lead to strong diagnostic suspicion. At this 

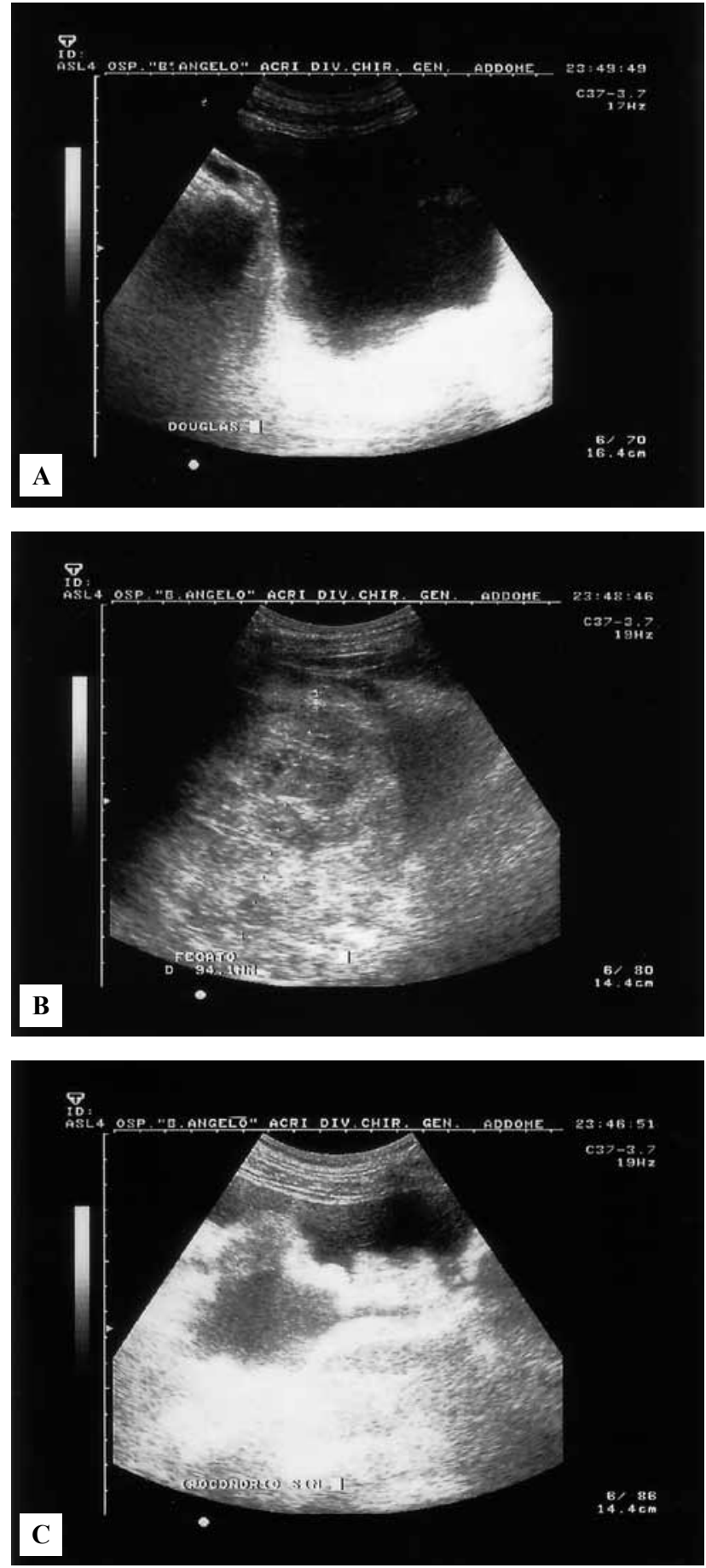

Fig. 1: US reveals hemoperitoneum

point it is necessary to address the therapeutic approaches. In patients with good liver function (Child-Pugh A-B7), the treatment of choice is liver resection. Otherwise, arterial embolization (TAE) $(8,11,12)$, is preferable in patients with advanced liver disease, poor functional hepatic reserve, or unresectable HCC. In this case the patient, before the rup-

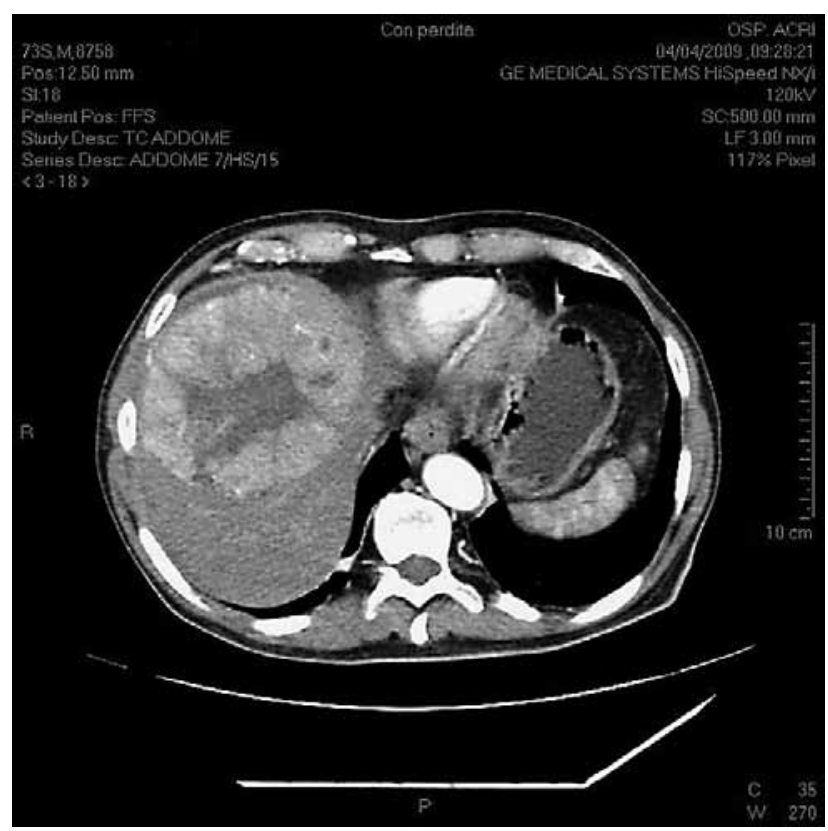

Fig. 2: CT scan performed 5 day before hospital admission, shows a very large $\mathrm{HCC}$, and free fluid around the liver

ture of HCC, had started therapy with sorafenib, at another hospital. Sorafenib is an inhibitor of the vascular endothelial growth factor (VEGF) pathway. In patients with advanced HCC, it causes an increase in survival and time to radiologic progression (13). Treatment with sorafenib is associated with a significant increase in the risk of bleeding (14). The role of VEGF on vessels and coagulation is not yet known. However, we know that VEGF is important for survival of endothelial cells and for maintaining the architecture and integrity of the vasculature (14). Therefore, inhibition of the VEGF pathway may lead to vascular damage and to the inability of vessels to repair after trauma, with consequent increased risk of bleeding (15). Therefore, sorafenib may increase the risk of bleeding and rupture of HCC in susceptible patients.

\section{References}

1. Cabibbo G, Craxì A. Epidemiology, risk factors and surveillance of hepatocellular carcinoma. Eur Rev Med Pharmacol Sci 2010 14:352-5.

2. Kim YI, Ki HS, Kim MH, et al. [Analysis of the clinical characteristics and prognostic factors of ruptured hepatocellular carcinoma]. Korean J Hepatol 2009; 15 148-58.

3. Vergara V, Muratore A, Bouzari H, et al. Spontaneous rupture of hepatocellula carcinoma: surgical resection and long term survival. Eur J Surg Oncol; 2000; 26: $770-2$.

4. Miyamoto M, Sudo T, Kuyama T. Spontaneous rupture of hepatocellular carcinoma: a review of 172 Japanese cases. Am J Gastroenterol 1991; 86: 67-71.

5. Yeh CN, Lee WC, Jeng LB, Chen MF, Yu MC. Spontaneous tumour rupture and prognosis in patients with hepatocellular carcinoma. Br J Surg 2002; 89: 1125-9.

6. Tan FL, Tan YM, Chung AY, Cheow PC, Chow PK, Ooi LL. Factors affecting early mortality in spontaneous rupture of hepatocellular carcinoma. ANZ J Surg 2006; 76: 448-52.

7. Kirikoshi H, Saito S, Yoneda M, et al. Outcomes and factors influencing survival in cirrhotic cases with spontaneous rupture of hepatocellular carcinoma: a multicenter study. BMC Gastroenterol 2009; 9: 29. 
8. Lai EC, Lau WY. Spontaneous rupture of hepatocellular carcinoma: a systematic review. Arch Surg 2006; 141: 191-8.

9. Choi BG, Park SH, Byun JY, Jung SE, Choi KH, Han JY. The findings of ruptured hepatocellular carcinoma on helical CT. Br J Radiol 2001; 74: 142-6.

10. Vivarelli M, Cavallari A, Bellusci R, De Raffaele E, Nardo B, Gozzetti G. Ruptured hepatocellular carcinoma: an important cause of spontaneous haemoperitoneum in Italy. Eur J Surg 1995; 161: 881-6.

11. Battula N, Madanur M, Priest O, et al. Spontaneous rupture of hepatocellular carcinoma: a Western experience. Am J Surg 2009; 197: 164-7.
12. Bassi N, Caratozzolo E, Bonariol L, et al. Management of ruptured hepatocellular carcinoma: Implications for therapy. World J Gastroenterol 2010; 16: 1221-5.

13. Llovet JM, Ricci S, Mazzaferro V, et al. Sorafenib in advanced hepatocellular carcinoma. N Engl J Med 2008; 359: 378-90.

14. Je Y, Schutz FAB, Choueiri TK. Risk of bleeding with vascular endothelial growth factor receptor tyrosine-kinase inhibitors sunitinib and sorafenib: a systematic review and meta-analysis of clinical trials. Lancet Oncol 2009; 10: 967-74.

15. Kamba T, McDonald DM. Mechanism of adverse effects of anti-VEGF therapy for cancer. Br J Cancer 2007; 96: 1788-95.

Received: $12 / 11 / 2010$

Corresponding author:

Accepted in revised form: 27/10/2011

Ferdinando Rombolà, via degli orti, 55 - 89861 Tropea (VV), Italy, 0039.(0)963.61364; e-mail: f.rombola@alice.it 